\title{
Three Level Heterogeneous Zonal Stable Election Protocol for Energy Efficiency in Wireless Sensor Networks
}

\author{
Abdelkader Benelhouri ${ }^{1,3, *}$, Hafida Idrissi-Saba ${ }^{1,3, * *}$, and Jilali Antarir ${ }^{2,3, * * *}$ \\ ${ }^{1}$ Faculty of Science, Ibn Zohr University, B.P. 8106, 80000 Agadir \\ ${ }^{2}$ Polydisciplinary Faculty, Ibn Zohr University, B.P. 271, 83000 Taroudant \\ ${ }^{3}$ Laboratory of Metrology and Information Processing(LMIP)
}

\begin{abstract}
Sensor battery limitation has always been the most challenging hurdle for wireless sensor networks. Many energy efficient routing protocols have been proposed to overcome this issue in homogeneous networks where sensor nodes start with the same initial energy. When sensor nodes have different amount of initial energy, the network is heterogeneous and it becomes complicated to design an energy efficient routing protocol to save nodes energy and prolong network lifetime. Herein, we propose a three level heterogeneous routing protocol to boost network stability period of wireless sensor networks. The network model splits up into five zones according to nodes initial energies and distance to base station. For data communication, the proposed model relies on two types of communications: Direct and Multi-Hop. The choice of the type of communication is made according to nodes initial energy and their distance to the base station. The clustering scheme is used just in the zones that contains nodes with higher energies. The simulation of our proposed scheme is done using Matlab simulator and the results are compared to the conventional heterogeneous routing protocols 3-level heterogeneous Stable Election Protocol and 3-level Modified Low Energy Adaptive Clustering Hierarchy.
\end{abstract}

Keywords. Heterogeneous routing potocol, threshold election, zonal protocol, election probability, network lifetime.

\section{Introduction}

A Wireless Sensor Network (WSN) is an ad-hoc network which sensors nodes are widely distributed in a region of interest for real-time data extraction. A sensor observes an event or collects physical data from its area of interest. Then it processes the observed phenomenon using a small integrated processor [1]. The sensor sends the processed data to a base station (BS). The sensor nodes act as both sensors and routing devices. Multiple sensor nodes can be used to transmit data to the destination (i.e, multi-hop communication).

When a WSN is deployed, each sensor has a limited amount of energy. Each action (i.e., detection, transmission, etc.) that is taken by a sensor has an energy cost that slowly exhausts the power of the sensor. The death of a single node does not have a major impact on the

\footnotetext{
*e-mail: a.benelhouri@gmail.com

**e-mail: h.idrissi_saba@hotmail.com

***e-mail: j.antari@uiz.ac.ma
} 
network, but when several nodes disappear, the performance of the network is reduced $[2,3]$. The WSN significantly expands our ability to monitor and control the physical environment from remote sites and improve the accuracy of information obtained through collaboration between sensor nodes and information at these nodes [1,4]. For this reason, WSNs are currently widely used in military, medical and commercial applications, etc.

The major challenge of a WSN is the limitation of sensors batteries. Sensors can not keep sending data for a long period of time [5,6]. Energy-Efficient routing protocols were and still the most important solution of the energy limitation issue [7]. When all sensors have the same amount of initial energy, the network is called homogeneous, in the other hand, when sensors have different amounts of initial energy, the network is heterogeneous. Many routing protocols were designed for homogeneous network, but in the case of an heterogeneous network, it becomes so difficult to design an Energy-Efficient routing protocol. In this paper we present a three level zonal stable election protocol for stability period boost and lifetime enhancement in heterogeneous WSNs. The performance of the proposed protocol is compared with ModLEACH (Modified Low Energy Adaptative Clustering Hierarchy), and hetSEP (Heterogeneous Stable Election Protocol)

\section{Literature review}

There are many challenges facing wireless sensor network, including efficient use of energy, security, scalability, robustness, connectivity and coverage [8]. Many routing protocols have been proposed to minimize energy consumption in homogeneous WSNs. In LEACH protocol [7], the network is divided into clusters with random selection of cluster heads, cluster members data are collected by the chosen cluster head and sent to the base station. Threshold sensitive Energy Efficient sensor Network (TEEN) protocol [9] saves network energy by reducing the number of transmissions. Sensor nodes could send data only if the detected feature value is equal or over the hard threshold. An improvement of LEACH protocol has been proposed by Lindsey and Raghavendra [10] and is known as power-efficient gathering in sensor information systems (PEGASIS protocol). In this model sensor nodes are organized to form a chain for data transmission. Each node receives data from its neighbor node, combines its own data with the received data and transmits it to the next node in the chain until the data reaches the base station. The hybrid energy efficient distributed (HEED) [11] clustering protocol is based on the estimation of residual energy of nodes as a primary parameter to select cluster heads, and node degree as secondary parameter which has the function of balancing energy load among all cluster heads. In SEP scheme [12] there are two energy levels and sensor nodes are divided into normal and advanced nodes. Due to their high cost factor, advanced nodes are less in number than normal nodes, but they have higher initial energy. SEP uses two weighted cluster-head election probabilities according to the type of node. A Distributed Energy Efficient Clustering (DEEC) protocol was proposed in [13] to prolong network lifetime in multi-level heterogeneous wireless sensor networks. The selection of $\mathrm{CHs}$ in DEEC is based on a ratio between the residual energy of each node and the average energy of the whole network. Every single node has its own probability to be a cluster-head. This probability is computed at each round based on residual energy of the node and the estimated average network energy of the current round. An improvement of DEEC protocol was proposed in[14] as: Threshold Distributed Energy Efficient Clustering (TDEEC) protocol. The main goal of TDEEC scheme was to enhance the stability period and improve energy efficiency of a given heterogeneous wireless sensor network. Each node has a specific threshold which includes a ratio between the residual energy of the node and the average energy of the whole network. in[15] a Geography-Based Heterogeneous Hierarchy Routing (GBHHR) 
protocol was proposed. GBHHR aims to improve data collection efficiency and solve the issue of cluster heads selection, this protocol uses a data filter function which helps to remove redundant data and save valuable related data. A modified LEACH protocol was proposed in [16]. The ModLeach protocol introduced an efficient cluster head replacement scheme by implementing hard and soft thresholds in cluster-head election process. At each round, if the current cluster head has less energy than the required threshold, it should be replaced by another cluster head.

\section{Proposed Scheme}

The network model contains $n$ sensor nodes and it splits into three types of nodes: $x$ normal nodes, $y$ advanced and $z$ super nodes. We assume that normal nodes are equipped with $E_{o}$ of initial energy, advanced nodes and super nodes owns $(1+\theta) \times E_{o}$ and $(1+\gamma) \times E_{o}$ respectively. In most heterogeneous routing protocols schemes, network nodes are distributed

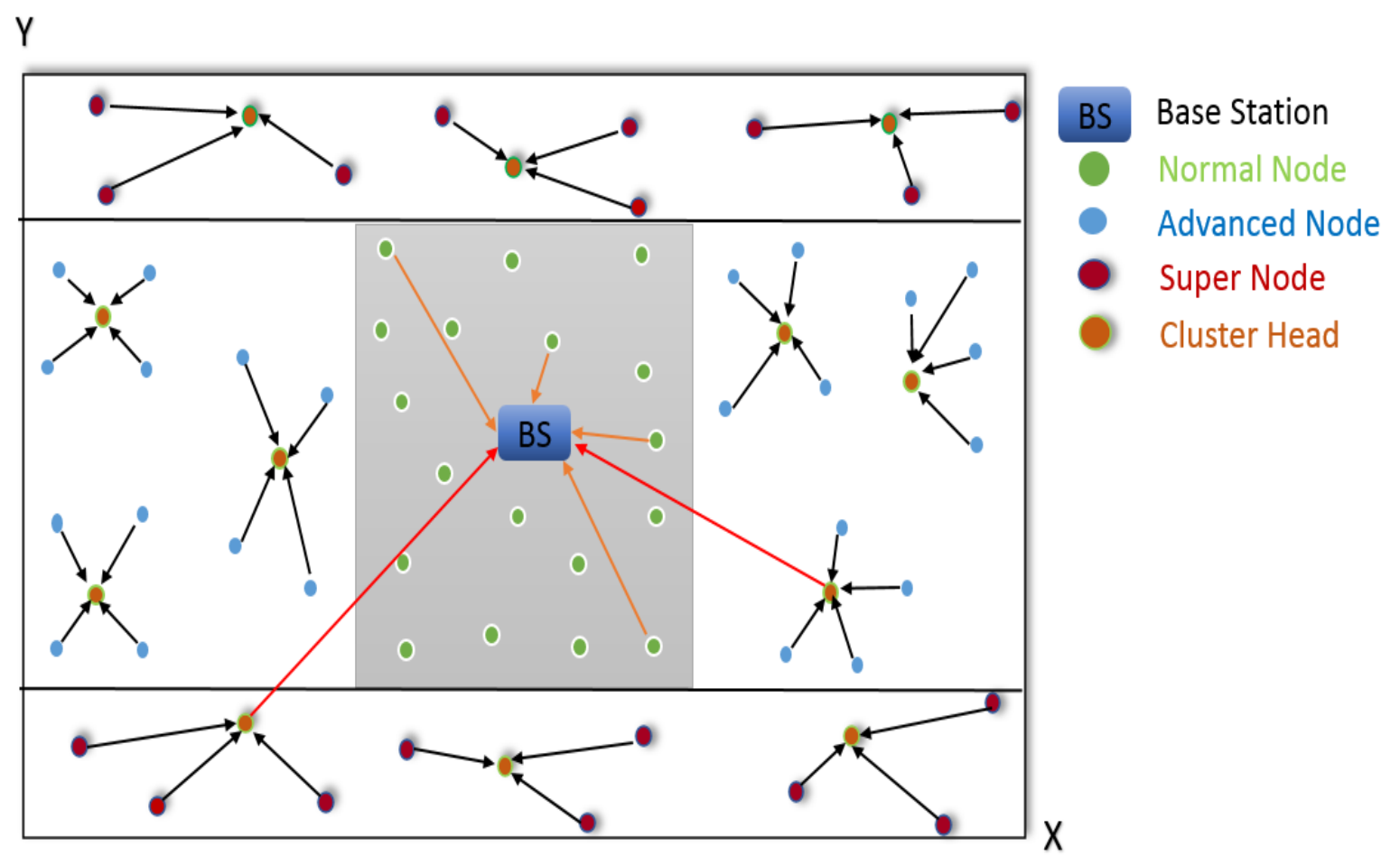

Figure 1. The proposed scheme nodes distribution.

randomly without consideration of the energy of nodes. In our proposed scheme, the distribution of nodes is based on nodes energy level and distance to the base station. Normal nodes are placed near the base station and they communicate their data directly because the transmission range is short and direct communication is more energy-efficient than multi-hop transmission. Advanced nodes are divided into two zones, they are located on the right and the left of normal nodes zone. Nodes with super energy level are placed at the top and bottom of the network. For data transmission, advanced nodes and super nodes are organized in clusters, at each cluster, a cluster-head $(\mathrm{CH})$ is elected to collect cluster members data and transmit it to the base station. The cluster-head selection process is based on weighted election probability which is computed based on nodes energy level. 
Table 1. Network parameters

\begin{tabular}{ll}
\hline Description & value \\
\hline$x$ Normal nodes count & 55 \\
$y$ Advanced nodes count & 30 \\
$z$ Super nodes count & 15 \\
$\theta$ Advanced nodes energy factor & $1.66 \mathrm{~J}$ \\
$\gamma$ Super nodes energy factor & $2.42 \mathrm{~J}$ \\
$\epsilon_{f s}$ Energy to transmit at a shorter distance & $10 \mathrm{~nJ} / \mathrm{bit} / \mathrm{m}^{2}$ \\
$\epsilon_{m p}$ Energy to transmit at a longer distance & $0.0013 \mathrm{pJ} / \mathrm{bi} / \mathrm{m}^{4}$ \\
$E_{\text {elec }}$ Energy consumed in the electronics circuit & $50 \mathrm{~nJ} / \mathrm{bit}$ \\
$E_{D A}$ Energy for data aggregation & $5 \mathrm{~nJ} / \mathrm{bit} / \mathrm{signal}$ \\
Network size & $100 \mathrm{M} \times 100 \mathrm{M}$ \\
Base station position & $(50 \mathrm{~m}, 50 \mathrm{~m})$ \\
Number of sensor nodes & 100 \\
$E_{0}$ Initial energy of a normal node & $0.5 \mathrm{~J}$ \\
$L$ Message size & $4000 \mathrm{bits}$ \\
\hline
\end{tabular}

The total initial energy of the 3-level heterogeneous network is given as below:

$$
\begin{aligned}
E_{t o t} & =x E_{o}+(1+\theta) y E_{o}+(1+\gamma) z E_{o} \\
& =E_{o}[x+(1+\theta) y+(1+\gamma) z]
\end{aligned}
$$

The probability threshold that each sensor node $S_{i}$ should depend on to decide itself as cluster head or not in each round is defined as follows:

$$
T\left(s_{i}\right)= \begin{cases}\frac{p_{i}}{1-p_{i}\left(r \bmod \frac{1}{p_{i}}\right)} & \text { if } S_{i} \in G \\ 0 & \text { Otherwise }\end{cases}
$$

$G$ is the set of nodes that are eligible to be cluster-head at the current round $r$.

Each type of node has its own probability to be cluster head as shown in the following equations:

$$
p_{i}= \begin{cases}\frac{p_{\text {opt }}}{x+(1+\theta) y+(1+\gamma) z} & \text { for normal nodes } \\ \frac{p_{\text {opt }}(1+\theta)}{x+(1+\theta) y+(1+\gamma) z} & \text { for advanced nodes } \\ \frac{p_{\text {opt }}(1+\gamma)}{x+(1+\theta) y+(1+\gamma) z} & \text { for super nodes }\end{cases}
$$

\section{Simulation and Results}

In this section, we evaluate the performance of our $3 L-Z S E P$ against $3 L-S E P$ and $3 L-M o d L E A C H$ protocols using MATLAB software. The network is 3-Level heterogeneous composed of 100 sensor nodes. The parameters of the network are shown in Table.1. The base station is located at the center of the monitored field. For data transmission energy consumption we use the first radio model described in SEP. Figure 1 shows the average lifetime of network. The network depletes all its energy in 4807, 5081 and 4812 rounds for 3L-SEP, 3L-ModLEACH and 3L-ZSEP. Mod-LEACH protocol guarantees the longest network lifetime, but in term of stability period, our proposed approach is better, $50 \%$ nodes survived for more than 2263 rounds for our 3L-ZSEP, 1788 rounds for 3L-SEP and 1379 
rounds for 3L-ModLEACH. For data transmission, our proposed protocol relies on 2 types of communications. For normal nodes zone, sensor nodes are so close to the base station, the transmission range is short, in this case, direct transmission consumes less energy than the transmission with clustering scheme. Advanced nodes and super nodes zones communicate their data through the elected cluster heads.



Figure 2. Alive nodes Vs. rounds.

The proposed strategy prevents normal nodes from exhausting their energy so quickly which has a major impact on network energy consumption rate as shown in figure 2 . Advanced and super nodes owns extra energy than normal nodes, it is not energy-efficient for the routing protocol to deal with normal nodes the way it deals with advanced and super nodes in data transmission process. To save network energy, normal nodes benefit from a short transmission range in comparison with advanced and super nodes. 3L-SEP and 3L-ModLEACH penalize normal sensors by letting them to transmit their data over a long distance which depletes their energy so fast and as a results, normal nodes survive for less rounds than our proposed protocol. The quick death effect of normal nodes is shown in the low throughput of 3L-SEP and 3L-ModLEACH as presented in figure 3. The proposed protocol prevents the quick death of normal nodes, these nodes can keep sending data for more rounds than other protocols, so the throughput of this protocol is extremely higher.

\section{Conclusion}

In this paper, the proposed protocol is designed to prolong the period stability and guarantee a great throughput in three-level heterogeneous wireless sensor networks. The network splits into five zones according to nodes initial energy. Data transmission is done using two types of communications in the aims of saving the lifetime of nodes with less energy. The proposed strategy has outperformed the 3L-SEP and the 3L-ModLEACH in terms of stability period and network throughput. 


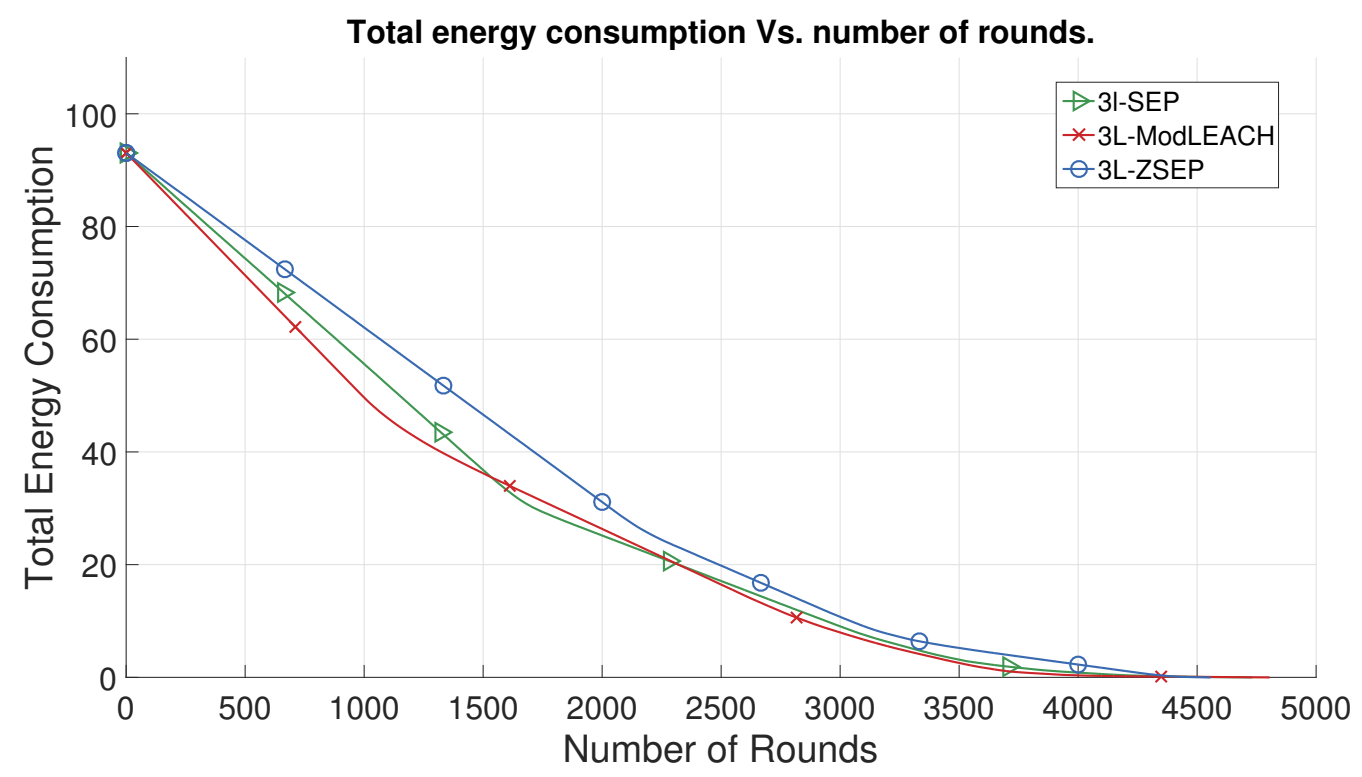

Figure 3. Energy Consumption Vs. rounds

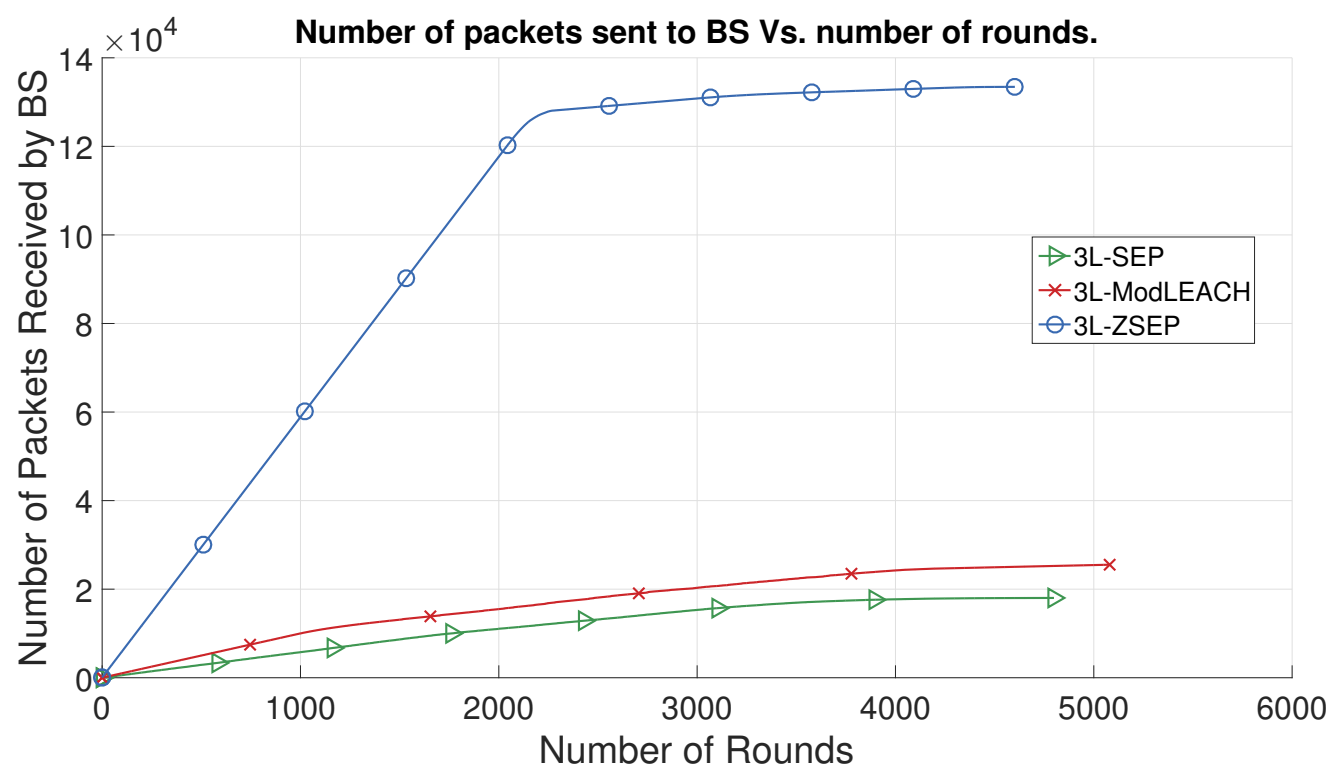

Figure 4. Packets received by the base station Vs. rounds

\section{References}

[1] I.F. Akyildiz, W. Su, Y. Sankarasubramaniam, E. Cayirci, IEEE Communications magazine 40, 102 (2002)

[2] N.A. Pantazis, S.A. Nikolidakis, D.D. Vergados, IEEE Communications surveys \& tutorials 15, 551 (2012)

[3] S. Lonare, G. Wahane, A survey on energy efficient routing protocols in wireless sensor network, in 2013 Fourth International Conference on Computing, Communications and Networking Technologies (ICCCNT) (IEEE, 2013), pp. 1-5

[4] N. Tadayon, S. Khoshroo, E. Askari, H. Wang, H. Michel, Journal of Network and Computer Applications 36, 1008 (2013) 
[5] K. Akkaya, M. Younis, Ad hoc networks 3, 325 (2005)

[6] T.D. Räty, IEEE Transactions on Systems, Man, and Cybernetics, Part C (Applications and Reviews) 40, 493 (2010)

[7] W.R. Heinzelman, A. Chandrakasan, H. Balakrishnan, Energy-efficient communication protocol for wireless microsensor networks, in Proceedings of the 33rd annual Hawaii international conference on system sciences (IEEE, 2000), pp. 10-pp

[8] J.N. Al-Karaki, A.E. Kamal, IEEE wireless communications 11, 6 (2004)

[9] A. Manjeshwar, D.P. Agrawal, TEEN: ARouting Protocol for Enhanced Efficiency in Wireless Sensor Networks., in ipdps (2001), Vol. 1, p. 189

[10] S. Lindsey, C.S. Raghavendra, PEGASIS: Power-efficient gathering in sensor information systems, in Proceedings, IEEE aerospace conference (IEEE, 2002), Vol. 3, pp. 3-3

[11] O. Younis, S. Fahmy, IEEE Transactions on mobile computing 3, 366 (2004)

[12] G. Smaragdakis, I. Matta, A. Bestavros, Tech. rep., Boston University Computer Science Department (2004)

[13] L. Qing, Q. Zhu, M. Wang, Computer communications 29, 2230 (2006)

[14] P. Saini, A.K. Sharma, International Journal of computer applications 6, 30 (2010)

[15] X. Chen, W. Qu, H. Ma, K. Li, A geography-based heterogeneous hierarchy routing protocol for wireless sensor networks, in 2008 10th IEEE International Conference on High Performance Computing and Communications (IEEE, 2008), pp. 767-774

[16] D. Mahmood, N. Javaid, S. Mahmood, S. Qureshi, A.M. Memon, T. Zaman, MODLEACH: a variant of LEACH for WSNs, in 2013 Eighth international conference on broadband and wireless computing, communication and applications (IEEE, 2013), pp. $158-163$ 